\title{
A Real-Time Intelligent System for Tracking Patient Condition
}

\author{
Filipe Portela ${ }^{1}$, Sérgio Oliveira ${ }^{1}$, Manuel Santos ${ }^{1}$, José Machado $^{2}$, António Abelha ${ }^{2}$ \\ 1,2 Algoritmi Centre, University of Minho, Portugal; \\ ${ }^{1}$ cfp@dsi.uminho.pt, sergiomdcoliveira@gmail.com,mfs@dsi.uminho.pt; \\ 2jmac@di.uminho.pt, abelha@di.uminho.pt
}

\begin{abstract}
Hospitals have multiple data sources, such as embedded systems, monitors and sensors. The number of data available is increasing and the information are used not only to care the patient but also to assist the decision processes. The introduction of intelligent environments in health care institutions has been adopted due their ability to provide useful information for health professionals, either in helping to identify prognosis or also to understand patient condition. Behind of this concept arises this Intelligent System to track patient condition (e.g. critic events) in health care. This system has the great advantage of being adaptable to the environment and user needs. The system is focused in identifying critic events from data streaming (e.g. vital signs and ventilation) which is particularly valuable for understanding the patient's condition. This work aims to demonstrate the process of creating an intelligent system capable of operating in a real environment using streaming data provided by ventilators and vital signs monitors. Its development is important to the physician because becomes possible crossing multiple variables in real-time by analyzing if a value is critic or not and if their variation has or not clinical importance.
\end{abstract}

Keywords: Ambient Intelligence, HealthCare, Data Streaming, Critic Events, Intelligent Systems, Real-Time, Tracking System, INTCare, Intensive Care

\section{Introduction}

The ability to extract useful knowledge from the data collected can provides better working practices tools [1]. Hospitals see in the database an important asset to the decision-making process [2]. The health sector is in constant development and evolution in order to provide better patient care and improving their services delivery. The technologies related to Ambient Intelligence has a great importance in providing health care, not only in the medical care field but also in the administrative and organizational field [3]. The use of appropriate tools by health professionals, can help them to identify causes and more effective treatments focused on patient disease [4].

This work aims to show the importance of developing a system capable of interacting with an intelligent environment system by combining the information needed by the clinician and the patient condition. This system is able to identify critic events for all monitored variables in two ways: detecting critic values by using a table containing the normal values range by attribute (minimum and maximum) and detecting variations in an attribute by analyzing the curve of values. The system was tested in a real environment - Intensive Care Unit (ICU) of Centro Hospitalar do Porto (CHP) and using the data provided by patient sensors: vital signs and ventilation. The 
achieved results by this new tool were very satisfactory. The intensivists see a big utility in using this tool. Their features help to quickly understand the patient condition more effectively. This tool is also able to send an alert when some abnormal value appears.

This article is divided in four sections. The first one shows the evolution of technology in health care and the work goals. The next chapter discusses and presents important aspects directly related to the development of this work. The third chapter presents the logical structure of the intelligent system developed. Then section four presents the main conclusions of the work and some guidelines for future work.

\section{Background}

\subsection{Ambient Intelligence, Intensive Care and Critic Events}

The Ambient Intelligence (AMI) corresponds to a future vision of intelligent computing in order to support people in various environments [5]. The primary focus behind the AMI environment is to enrich a technology particularly with the use of sensors and devices capable of communicating with database systems. Thus, you can build systems able to provide solutions to the user benefit based on the actual perception and historical information, habits and even emotions [6][7]. AMI promotes successful interpretation of contextual information from sensors and can adapts it to user needs in a transparently and proactively way. Due AIM features the ICU of CHP [17]is an example of AMI.

Patients who are in an ICU are continuous monitored, in weak health condition and in a serious life-threatening [8]. The patient care in this unit has several adverse events. The respective events can be factors of great impact in the clinical condition of the patient in the future [9]. These events can occur several times a day. The possibility of having the ability to identify events automatically and in real-time represents an important help to the decision process [10]. Patient condition can modify several times for hour. To an Intensivist is very difficult to analyze and compare all the values in realtime. The system developed take off this concern from the intensivist giving to them more time to care the patient. The system is able to analyze all the values received and in real-time notify when some abnormal situation appears. Several studies have been conducted showing that the most common adverse errors are related to the mechanical or human error [11]. However, there are other problems that are difficult to analyse, for example clinical events. Usually these events are identified from some guidelines supplied by bedside monitors [12] and data streaming (e.g. vital signs and ventilation).

The identification of a critic event is recognized when an attribute values is out of the normal range for a certain period of time [13]. An earlier study was made in order to track vital signs critic events based in the value collected and the time event [10]. The results achieved in the past were the first step to develop a universal and most complete system by giving the possibility to the user configure it.

\subsection{INTCare}

This work was carried out under the research project INTCare. INTCare is an Intelligent Decision Support System (IDSS) [14] designed to ICUs. INTCare allows continuously 
monitoring of the data collected by the sensors connected to the patient. At same time this IDSS is able to predict clinical events in real-time by using data mining and the data collected. One of its goals is to make their job in a pervasive way $[15,16]$. INTCare [18] was an important trigger to develop an intelligent environment. The environment is now prepared to identify critic events and support the system developed in real-time.

\section{Tracking System}

This is an intelligent and autonomous system, where the decision makers can use it to track patient values in two ways: analyze if a value is too low or too higher to be considered critic; analyze if the value variance is significant and if it has some clinical significance. In the first case (algorithm 1) an intelligent agent is used to categorize all the values collected in real-time. The agent categorizes the value received as 0 (not critic) or 1 (critic) using the table containing the ranges. For example if the SPO2 value is critic when the value is lower than 80 , always a value lower than 80 arises, the system identifies it as critic. Then the critic events concept already used in intensive medicine [10] is applied. In the second case (algorithm 2) an agent is used to monitoring the variation of values by variable. The user can define a clinical significance in order to better understand the curve values. For example if the user defines a Blood Pressure with a variation from the last value upper than 20 , it should be considered to future analysis. In this case the system indicates when the defined variance is verified. Additionally the user also can define a time. For example if the values collected had those variation for a time duration of 10 minutes, it can be considered as a clinical event.

\subsection{Acquisition Process and Data Selection}

The system can be adopted to different fields of study. In this case it was used for looking by critic values in several patient ventilation and vital signs atributes. In both cases the data inserted into the database are uninformative and difficult to understand. In this case it was necessary to carry out actions in order to demonstrate their value to doctors or nurses. This tracking system is designed to answer in real-time to the user needs or to alert when some abnormal value is collected. During the acquisition process a trigger is executed to assess if the value is valid and how critic is it. Then the second part of the system is executed by an intelligent agent. This agent is executed periodically. First it analyses the variation and then in accordance to the user indications it can analyze the clinical significance of the values collected.

The system developed is mainly concerned with the identification of critic events and to identify their significance. In the first case the table with the ranges is used to identify if a value is critic. In the second case critic events are identified only due to a changing of value. The table containning the range of values must be determined based on scientific and clinical knowledge by the hospital service / unit. Attributes can have several changes in values, however it is chosen the variations that best meets the enduser needs. In both cases the identification of the attributes and their underlying changes in the value are fundamental to a properly identification of the events. 


\subsection{Data Processing}

The system automatically categorize a value as critic or not, covering patient streaming data. These records are used to determine their criticity, just in case if they have not been previously used. The number of rows is depending from the number of admitted patients in the service. After a patient be discharged all the data is transferred to other table. Periodically it is executed another agent to verify if all the records were processed. In fact there is a temporal spectrum to view critic events. The time range may be, for example, corresponding to records up to the last two hours.

Algorithm 1 presents the trigger to identify if a value collected is valid and then if it is critic or not. Critic value table contains the range of possible values and the normal values. If a patient has a valid value (inside of the normal range) and a value is lower than a minimum or it is higher than a maximum, the value collected is considered critic. The critic table is defined by the clinical staff according to their environment and type of patients. By default a table defined by some experts in the field [10] is used. In this case and according to the critic table, a value can be normal (0), critic (1) or too critic (2). This process is executed always some new value arrives.

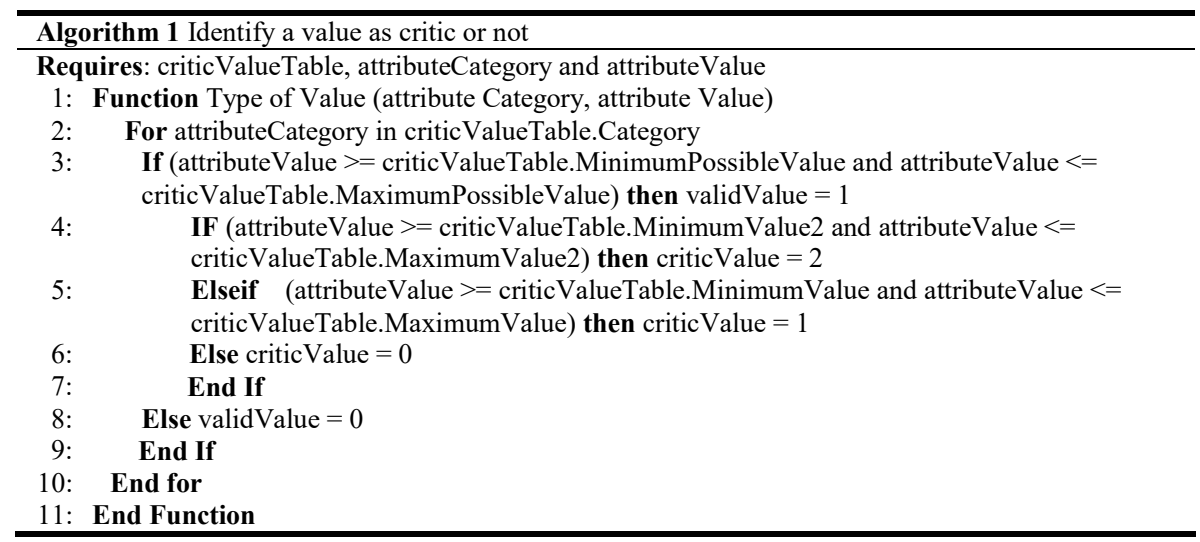

Algorithm 2 is used to identify possible changes in the values collected, i.e., if the value now received is different than the value earlier received. The procedure responsible for carrying out the identification of critic events is capable of performing two more operations: changing the count values of the attributes and performing a magnitude analysis of the values difference.

The two next steps are made following the records timeline using an ascending order by attribute and patient identification. The counting process changes gives to the health professional the possibility to follow the patient and the number of value changes of a particular attribute monitored during a certain period of time. This information is useful to the health professionals because it extends their capability in identifying the patient condition and stability.

The ability to identify the difference of values allows the professionals to identify how sharp are being the values variations. Professionals also can identify how long a value is keeping stable. This information can be useful to for example modifying a ventilation parameter or to define a new therapeutic. In order to understanding these operations, the following algorithmic representation presents the system operations. 


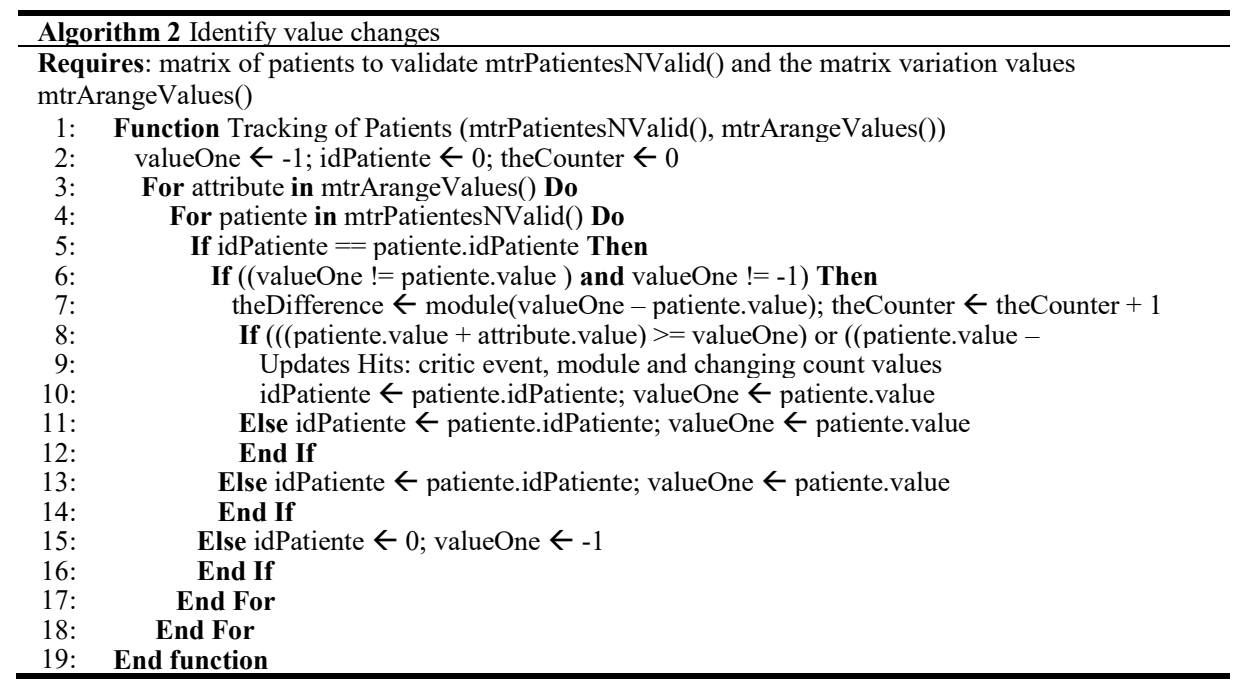

Being the system responsible for feeding the main repository, it performs various operations on the data collected. If this operation is not implemented the time responses to professional requests would sequentially increase. Thus the information presented is always timely and focused on patients who are admited in the service. If a professionals wants to consult earlier data, the system will use the historical repository.

\subsection{Clinical benefits}

The system developed enables determining value changes and identifying critic events. The changes made by the system about the events allows the health professionals knowing how long a patient is having a critic event and how much the values varies. For example and using the ventilation system, this solution can be used to support the patient weaning process by analyzing the evolution of the associated variables. This system also gives the possibility to send alerts to the professionals with the most relevant results. The system is always running in background and when some of the conditions above mentioned appears the professional receives a message with the values and their changes. It allows to have a pervasive system, where the user did not need to be concerned with the system because it is prepared to always alert / inform when some abnormal situation is verified.

\section{Conclusion and Future Work}

Once completed the development of this intelligent system, their implementation in real environment (ICU of CHP) was important to identify the strengths and weakness of the system. During this process it was easy to understand their utility to health professionals in order to provide better patient care. As strengths they mentioned that the system is able to quickly identify critic events for all patients who are monitored by vital signs monitor or ventilator. It is easy to change system parameters, respectively the ranges of 
values that represent the critic events. Thus health professionals have the freedom to make their independent research. As a possible aspect of evolution it is the using of these data to predict critic values and curve variations. Although this system been designed inside a specific unit (ICU), it also can be transposed to other services / units and can be used in telemedicine and Ambient Intelligence without any changes.

For future work should be taken into consideration the following aspects: complementing the system with DM models to predict critic events and patient diseases; predecting future values by analyzing the curve; addressing and anticipating significant features in intelligent environment and apply the system to other services.

Acknowledgments. This work has been supported by FCT - Fundação para a Ciência e Tecnologia within the Project UID/CEC/00319/2013 and PTDC/EEI-SII/1302/2012.

\section{References}

[1] M. Santos and C. Azevedo, Data Mining Descoberta do conhecimento em base de dados. FCA - Editora de Informática, Lda, 2005.

[2] Portela, F., Santos, M. F., Gago, P., Silva, Á., Rua, F., Abelha, A.,. \& Neves, J.. Enabling real-time intelligent decision support in intensive care. ESM 2011. (2011).

[3] F. A. S. Marins, R. Rodrigues, F. Portela, M. F. Santos, A. Abelha, and J. M. Machado, "Extending a patient monitoring system with identification and localisation," 2013 IEEM, Dec. 2013.

[4] H. Koh and G. Tan, "Data mining applications in healthcare," J Healthc Inf Manag, vol. 19, no. 2, pp. 64-72, 2005.

[5] G. Acampora, D. J. Cook, P. Rashidi, and A. V. Vasilakos, "A Survey on Ambient Intelligence in Healthcare," Proceedings of the IEEE, vol. 101, no. 12, pp. 2470-2494, Dezembro 2013.

[6] J. C. Augusto, "Ambient Intelligence: Basic Concepts and Applications," in Software and Data Technologies, J. Filipe, B. Shishkov, and M. Helfert, Eds. Springer Berlin Heidelberg, 2008, pp. 16-26.

[7] J. M. Machado, A. Abelha, J. Neves, and M. Santos, "Ambient intelligence in medicine," IEEE BioCAS 2006, art. no. 4600316, pp. 1-4, 2006.

[8] N. Bricon-Souf and C. R. Newman, "Context awareness in health care: a review," Int J Med Inform, vol. 76, no. 1, pp. 2-12, Jan. 2007.

[9] J. M. Rothschild, C. P. Landrigan, J. W. Cronin, R. Kaushal, S. W. Lockley, E. Burdick et. al., "The Critic Care Safety Study: The incidence and nature of adverse events and serious medical errors in intensive care," Crit. Care Med., vol. 33, no. 8, pp. 1694-1700, Aug. 2005.

[10]F. Portela, P. Gago, M. F. Santos, J. M. Machado, A. Abelha, Á. Silva, and F. Rua, "Implementing a Pervasive real-time intelligent system for tracking critic events in intensive care patients," IJHISI, 2013.

[11] M. Kaur, M. Pawar, J. K. Kohli, and S. Mishra, "Critic events in intensive care unit," Indian J Crit Care Med, vol. 12, no. 1, pp. 28-31, 2008.

[12]M. T. Keegan, O. Gajic, and B. Afessa, "Severity of illness scoring systems in the intensive care unit," Crit. Care Med., vol. 39, no. 1, pp. 163-169, Jan. 2011.

[13] A. Silva, P. Cortez, M. F. Santos, L. Gomes, and J. Neves, "Rating organ failure via adverse events using data mining in the intensive care unit," Artif Intell Med, vol. 43, no. 3, pp. 179-193, Jul. 2008.

[14]F. Portela, M. F. Santos, J. Machado, A. Abelha, Á. Silva, and F. Rua, "Pervasive and Intelligent Decision Support in Intensive Medicine - The Complete Picture," in Information Technology in Bioand Medical Informatics, Eds. Springer International Publishing, 2014, pp. 87-102.

[15]Portela, F., Santos, M.F., Vilas-Boas, M.: A Pervasive Approach to a Real-Time Intelligent Decision Support System in Intensive Medicine. In: Springer (ed.) CCIS, vol. 0272, pp. 14 (2012)

[16] Portela, F., Gago, P., Santos, M.F., Silva, A., Rua, F., Machado, J., Abelha, A., Neves, J.: Knowledge Discovery for Pervasive and Real-Time Intelligent Decision Support in Intensive Care Medicine. KMIS 2011, pp. 12. Paris, France (2011).

[17] Santos, M. F., Portela, F., Vilas-Boas, M., Machado, J., Abelha, A., Neves, J.,. \& Rua, F.. Information architecture for intelligent decision support in intensive medicine. Transactions on Computers. (2009).

[18] Filipe Portela, Jorge Aguiar, Manuel Filipe Santos, Álvaro Silva, Fernado Rua: Pervasive Intelligent Decision Support System - Technology Acceptance in Intensive Care Units. In: Springer AISC (2013) 